\title{
OPINIÓN \\ Educación integral \\ hace jóvenes aptos para enfrentar el mundo actual
}

Fundación Salvador del Mundo, Fusalmo

Recibido: 05/03/2014 - Aceptado: 21/04/2014

\section{Resumen}

El interés de abordar la educación como una herramienta para prevenir la violencia surge a partir de la experiencia del trabajo educativo que realiza la Fundación Salvador del Mundo (Fusalmo) con jóvenes que se encuentran en condiciones de riesgo y limitaciones socioeconómicas. Mucho se habla acerca del papel que la educación juega en el desarrollo de las personas, pero poco se dice del impacto que llega a tener, ya que la educación es más que transmitir conocimientos nuevos a una persona.

Fusalmo, desde hace más de diez años, apuesta al Programa Integral Juvenil Don Bosco (PIJDB), que constituye una oferta educativa complementaria al sistema educativo formal del Ministerio de Educación, ante las limitaciones experimentadas en localidades importantes por su densidad poblacional y la problemática de violencia amenazante, de manera que se orienta a población juvenil estudiantil que se encuentra amenazada por la violencia en sus comunidades y que vive en condiciones de vulnerabilidad económica y social. El Programa se desarrolla en los tres polideportivos Don Bosco, en las principales ciudades del país (San Miguel, Santa Ana y Soyapango).

\section{Palabras clave}

Educación, Educación-Aspectos sociales; Educación y delincuencia - Prevención del delito.

\section{Abstract}

The interest of addressing education as a tool to prevent violence arises from the experience obtained through the educational work carried out by the Fundación Salvador del Mundo (Salvador del Mundo Foundation, FUSALMOgiven its Spanish acronym) with young people who are at risk and live under serious socio-economic constraints. A lot has been said about the role played by education in the development of a country, however, little has been stated about its impact. After all, education is much more than merely transmitting new knowledge to a person.

More than ten years ago, FUSALMO decided to trust the Programa Integral Juvenil Don Bosco (Don Bosco's Youth Integral Program--PIJDB, given its Spanish acronym) which turned to be a complementary educational offer to the formal educational system offered by the Ministry of Education, in the face of the numerous limitations experienced in major towns with a high population and an increasing violence rate. It is targeted to the teenage student population threatened by the amount of violence in their communities and who live under vulnerable economic and social conditions. The program is currently developed at Don Bosco's three sports centers located in the country's major cities (San Miguel, Santa Ana and Soyapango).

\section{Keywords}

Education, Education-Social aspects; Education and delinquency - Crime prevention. 


\section{Responsabilidad de garantizar el derecho a la educación a la niñez y la juventud}

Siendo el Ministerio de Educación la entidad del Estado responsable de garantizar el derecho a la educación a la niñez y juventud en el país, el PIJDB complementa las acciones correspondientes a la escuela, como la educación física, la educación tecnológica y la educación en cultura de paz atención a la familia. De manera que el programa contribuye así, desde el enfoque de los derechos humanos y el carisma salesiano, a través del sistema preventivo, a brindar educación en sectores donde las escuelas tienen limitaciones en su infraestructura, sus espacios y de recursos que no les permite desarrollar todas las áreas de formación importantes para el desarrollo de la niñez y la adolescencia.

Fusalmo tiene claro que la educación no debe ser una tarea dedicada exclusiva a la transmisión de conocimientos, sino encaminada a la formación para la vida, a través del enriquecimiento actitudinal y espiritual, de dotar de herramientas a los jóvenes, que les permita tomar decisiones oportunas para su desarrollo personal y social en medio de sus contextos particulares y en riesgo. Es por ello que el enfoque general de la Fundación es brindar un espacio de atención integral en donde la niñez y la adolescencia, pertenecientes a la educación formal, desarrollen competencias técnicas, sociales y personales que les permita mejorar su calidad de vida con carisma salesiano.

Para identificar el exitoso camino de este programa, así como otros proyectos y programas ofrecidos por Fusalmo durante estos años, es necesario entender el carisma salesiano desde la realidad de los jóvenes salvadoreños. San Juan Bosco, llamado Don Bosco —un sacerdote comprometido con la juventud-, en el siglo XIX crea el sistema preventivo como método educativo para "prevenir" en base a la razón, religión y el amor a los jóvenes de los riesgos a sus alrededores y para invitarlos a crear un plan de vida como "Buenos cristianos y honrados ciudadanos". Un enfoque diferente del acercamiento que el educador debería tener hacia sus estudiantes es clave para el desenvolvimiento de estos; dejar de lado el castigo y, por supuesto, los golpes además de las palabras hirientes para señalar actitudes positivas de liderazgo, esfuerzo y disciplina, garantía al fin de cuentas de éxito en sus vidas.
En el entorno actual de los jóvenes con que trabaja Fusalmo, donde existen dificultades para acceder al estudio o empleo de calidad, carecen de posibilidades concretas para que construyan proyectos de vida claros y se ven amenazados constantemente por la violencia. Los espacios de formación juvenil deben responder siendo además de fuente de conocimiento, lugares de convivencia, que promuevan la participación del joven y se perciba en un ambiente de familia en la que se sienta apreciado. Ello es lo que se reflexiona en este artículo, en qué medida de lo posible los procesos educativos pueden ser mecanismos donde el aprendizaje no es cognitivo únicamente, sino que prepara al joven para convivir con los demás y desenvolverse en su entorno.

Se espera, por tanto, comprender la importancia que tiene el acercamiento al joven de quien educa, y que la generación de aprendizajes significativos en su vida se logra en la medida que los procesos educativos y sus recursos se ponen en función de los jóvenes y sus necesidades, principalmente para aquellas personas o instituciones que son responsables de la implantación de este tipo de experiencias.

Fusalmo, para identificar aquellas necesidades enfrentadas por los jóvenes atendidos, realizó una investigación durante el 2013 gracias al Departamento de Investigación y Desarrollo, la cual consistía en utilizar una metodología cualitativa, a fin de rescatar y reconstruir la experiencia de los jóvenes participantes en el PIJDB acerca de los impactos favorables que han alcanzado a través del proceso formativo vivido. En primer lugar identificamos personas clave en la implantación de dicho programa, quienes formaron parte activa en el proceso de consulta para recoger la información requerida, tales como jóvenes estudiantes entre 14 a 18 años edad, estudiantes de tercer ciclo de distintos centros escolares públicos y residentes en el municipio de Soyapango, madres de familia, educadores y educadoras de Fusalmo así como sus jefaturas.

Un grupo focal se utilizó para recibir los aportes de la población juvenil atendida en el programa educativo de Fusalmo, y además de las madres de familia que también participan en el mismo programa educativo. Así también se realizaron entrevistas individuales para consultarle a personal clave en la realización del programa educativo, que por la experiencia y el papel desempeñado sus aportes son valiosos para enriquecer los análisis sobre la influencia 
positiva que tiene el programa en la prevención de la violencia en los jóvenes.

Como parte de los resultados, frecuentemente se habla de la educación como uno de los principales recursos de las sociedades para mejorar su nivel de productividad y mejora de la calidad de vida. Sin embargo, en la experiencia formativa que Fusalmo permite evidenciar, que la educación, más allá de ser un cúmulo de conocimientos, implica una dimensión sumamente humana que contribuye en mejorar las relación de las personas con su entorno. Claro está, que hacer resaltar esa dimensión humana depende en gran mucha medida del enfoque educativo que se utilice za para intercambiar conocimientos y experiencias entre las personas involucradas en la experiencia de aprendizaje.

Una característica primordial en la implantación de este programa educativo es la espiritualidad salesiana de la práctica educativa. El enfoque salesiano da un soporte espiritual a la práctica educativa en el programa. Esto significa que, desde la mística salesiana, el papel de la persona responsable de la formación no es únicamente enseñar contenidos, sino dar un acompañamiento al joven, permitirle que sea protagonista de su propio crecimiento, hacerle sentirse acogido y destacar en él todo lo positivo que pueda tener.

Precisamente esto es uno de los principales fundamentos del enfoque educativo que propone Fusalmo, que la educación debe ser integral, que atienda la dimensión humana de los jóvenes, que fortalezca su espiritualidad como personas y que los aliente a ser protagonistas de su desarrollo en medio de su contexto de vida, aun cuando este pueda ser adverso. Estos aspectos, en conjunto, hacen que la educación se convierta en un recurso para prevenir la violencia.

Fusalmo brinda una formación complementaria a la oferta del sistema formal, dentro del proceso educativo se enfoca fundamentalmente en las áreas educativas de: tecnología, cultura de paz, orientación vocacional y educación física. ¿Por qué estas áreas en particular? Estas responden a las necesidades y demandas de formación de la población juvenil en los contextos ya descritos, y además son áreas educativas que los centros escolares públicos no logran desarrollar debido a las limitaciones de infraestructura y recursos que adolecen.
Tomando estas áreas educativas en el programa, se investigó con los jóvenes - estudiantes de tercer ciclocuáles son los aprendizajes que logran consolidar en la formación recibida. Estos hallazgos resultan interesantes, ya que muestran los alcances que puede tener una educación orientada en el fomento del protagonismo de los jóvenes e interesada de genuinamente en su persona.

Es por ello que se identificó, en base a las necesidades de los jóvenes, que en el área de tecnología se generan aprendizajes básicos en el uso de las herramientas tecnológicas; y evidencian la utilidad de estas herramientas para aprovecharlas en su vida diaria. Chicos que jamás habían tocado una computadora, o incluso nunca la habían visto, hoy tienen esa oportunidad de tener acceso al equipo, incluso a trabajar en internet las tareas de la escuela. Otra de las acciones es aprender a formarse una proyección de vida, siendo al final uno de los logros rescatados de la experiencia de estos jóvenes, que se han alcanzado mediante la orientación vocacional recibida.

En el área de educación física y deporte, se complementan los elementos que no se logran abarcar desde la escuela: los jóvenes aprenden sobre sus habilidades físicas y deportivas al mismo tiempo que adquieren consciencia sobre la importancia del trabajo en equipo. Fusalmo, en las instalaciones de los polideportivos Don Bosco, busca brindar a estos chicos amplias instalaciones en donde pueden correr, recrearse, saltar, incluso jugar a las escondidillas durante los recreos mientras que en sus centros escolares deben evitar chocar con otros.

Otra de las áreas educativas más significativas según los testimonios de los jóvenes es cultura de paz, el cual brinda los fundamentos para sensibilizar a los jóvenes en la prevención de la violencia y en el fomento de una convivencia pacífica. Para ello, los aprendizajes destacados están orientados a que los jóvenes identifiquen las expresiones de violencia y a ser más protagonistas de su desenvolvimiento y en su relación con su entorno.

Es importante recalcar que la estrategia educativa de Fusalmo, para implantar el PIJDB, le apuesta a desarrollar una práctica integral en la atención a los jóvenes y prevenir la violencia, así se reafirma que además del aprendizaje de nuevos conocimientos se alcance la incidencia en 
habilidades y actitudes en los jóvenes. Estos hallazgos ayudan a comprender que un proceso educativo que procure atender diversas dimensiones en los jóvenes tendrá mayor oportunidad de generar cambios cognitivos, de capacidades y actitudinales.

\section{Impactos en la vida cotidiana de los jóvenes}

Después de observar los aprendizajes que se generan en la población atendida, a partir de sus propias experiencias, se identificaron los impactos fundamentales que son alcanzados por los jóvenes atendidos en el PIJDB. Estos impactos son relevantes, ya que sus alcances van más allá del programa, incidiendo al interior de la familia y en el centro escolar como sus principales entornos de vida.

De manera que, mediante la aproximación a la experiencia de los jóvenes consultados, han definido los impactos generados mediante el proceso formativo vivenciado: identificamos que existe una mejora en la valía personal del joven. Un impacto que tiene gran predominancia en las opiniones de los jóvenes es haber aprendido a valorarse más a sí mismos, a sentir amor propio y sentirse personas capaces de lograr cosas para su vida. Consideran que eso les ha servido para enfrentarse de mejor manera a su propia realidad, evitar hacer acciones de riesgo y establecer relaciones inadecuadas con las personas que los rodean.

En el entorno que se desenvuelven estos jóvenes, es sumamente importante fomentar la autoestima, ya que esta es un recurso que les permite hacerle frente a las condiciones desfavorables que muchas veces experimentan. Otro detalle descubierto es consciencia de su realidad. Los jóvenes expresan ser más conscientes de la realidad que los rodea y de la importancia que tiene la historia en el desarrollo de la sociedad. Es una visión innovadora para muchos jóvenes, ya que les ayuda a comprender en mejor forma la sociedad en la que viven e interaccionar más adecuadamente con ella. Resulta interesante que los jóvenes relacionan que, tal como la sociedad tiene una historia, las personas tienen una historia personal vinculada con su familia, de manera que alcanzan una mejor comprensión del porqué de las situaciones que vive su familia.

\section{Mejora de habilidades para la convivencia}

Los jóvenes consultados destacan haber aprendido a comunicarse de una forma diferente a lo acostumbrado, expresando sus ideas y sentimientos más clara y adecuadamente; al interior de la familia, hacer el esfuerzo por escuchar a los demás. Otro elemento clave es la sensibilización hacia las manifestaciones de violencia y la concientización de que puede asumirse una actitud en contra de ella y actuarse de forma pacífica. Otro aspecto es mayor liderazgo y protagonismo en la interacción con su entorno. Mayor seguridad en sí mismos para desenvolverse y desempeñarse en el centro escolar y en la comunidad. Fortalecen su papel como líderes en los grupos en los que se integran, mejorar la capacidad de trabajar en equipo en actividades académicas y ser menos influenciables por los patrones violentos en sus comunidades.

La experiencia en la formación juvenil de Fusalmo la Fundación Salvador del Mundo fundamentada en el carisma salesiano, ha llevado a constatar que la educación, tanto para la niñez como para los jóvenes, es un recurso elemental para fomentar su desarrollo y como una fuente que brinda herramientas para prevenir la violencia. Sin embargo, la transmisión de información entre los educadores y los jóvenes por sí misma no es garantía de que los jóvenes aspiren a un mayor desarrollo y se sensibilicen para evitar ser víctimas o victimarios de otras personas en su vida cotidiana. La práctica educativa se enriquece en la medida que con los jóvenes se toman en cuenta tres elementos clave desde la experiencia de Fusalmo:

\section{a. La formación como una respuesta oportuna a las necesidades de los jóvenes}

Es esencial que los procesos educativos que se implantan con la población juvenil sean adecuados a las necesidades formativas que tienen los jóvenes. De manera que no sean los jóvenes quienes se adecúan al programa, sino que este se construye en función a sus demandas, solo así este se convertirá en una respuesta efectiva a la realidad en la que viven los jóvenes. Precisamente una dimensión de esa realidad es la manifestación de violencia que predomina en sus ambientes de vida, la cual debe de ser confrontada tanto con los contenidos que se le brindan como con las orientaciones de los educadores.

\section{b. La práctica educativa tiene a la base el acompaña- miento de brinda el educador}

El acompañamiento consiste en la proximidad al joven, en el sistema preventivo; significa orientar una dirección, 
consejo espiritual y humano para el joven; la promoción del asociacionismo juvenil; darle seguimiento a las acciones que realizan los jóvenes y la construcción de ambientes educativos sanos y familiares para el joven.

La figura del educador debe ser un elemento fundamental en el acompañamiento dado al joven, ya que este se constituye en un modelo para aquel. Es decir, que el educador -la persona responsable de la formación— más que un docente que transmite conocimientos debe ser una persona que, a través de su comportamiento, sus actitudes y su auténtico interés por el joven sea capaz de contagiarle el interés por crecer como persona y de convivir más pacíficamente con los demás.

\section{c. El papel protagónico que promueve al joven en su experiencia de aprendizaje}

El esquema del docente (el que sabe) frente a los estudiantes (los que aprenden) es una forma educativa que limita las capacidades de los jóvenes, vuelve la experiencia de aprender como algo tedioso; iy eso lo lleva a asumir un papel pasivo en su propio aprendizaje! La experiencia educativa debe ser constructiva para el joven no solo a nivel cognitivo, sino afectivo y relacional, es decir, que se le debe ayudar a que mediante la formación recibida descubra y potencie sus capacidades intelectuales, de afectividad y de relacionarse con las demás personas, eso permitirá que se apropien de su aprendizaje.

¿Cómo se alcanza ese protagonismo? En la medida que la formación es interesante porque atiende a sus necesidades, cuando los contenidos se transmiten mediante acciones que los hace partícipes y pueden cuestionar, aportar y enriquecer el mismo contenido y en la medida que se estimula la convivencia con sus demás compañeros y se promueve un trabajo más en equipo y menos competitivo.

\section{Impactos que se pueden promover en la educación en los jóvenes}

La investigación realizada por Fusalmo identifica impactos en los jóvenes que, como se ha evidenciado, no son impactos "académicos", sino que consisten en alcances que los benefician en su vida familiar, social, afectiva y espiritual. Esto permite recatar que así como en el PIJDB existen procesos educativos que procuran incidir en el joven en todas sus dimensiones, no únicamente en el enriquecimiento de sus conocimientos.

Desde el enfoque y experiencia de trabajo de Fusalmo se tiene la noción de que la educación debe permitir que el joven identifique sus propias capacidades y se sienta capaz de potenciarlas, que puede asumir un papel más protagónico en su propio desarrollo de vida y ser más consciente de lo que le rodea. En la medida que se promuevan estos logros en los jóvenes, se estará respondiendo a las necesidades que tienen y se les estará preparando para afrontar el contexto de violencia del que viven rodeados e amenazados.

Es evidente que erradicar la violencia es una meta demasiado compleja para procesos educativos complementarios en los jóvenes. Sin embargo, es posible y necesario que esta educación les permita a los jóvenes convertirse en actores de cambio en sus entornos de vida en los que se desenvuelven, para erradicar las manifestaciones de violencia que ellos generan o sufren y para promover una convivencia más pacífica en las relaciones que establecen.

La educación es una herramienta sumamente valiosa para prevenir la violencia, sobre todo cuando aborda las diversas esferas de la vida de los jóvenes. Precisamente ahí radica la importancia de los elementos clave que se han descrito previamente. Así se cumple el propósito educativo de que los conocimientos construidos además de ser un cúmulo de ideas, se constituyan en herramientas útiles para que el joven se desenvuelva de forma pacífica en su entorno, reconozca su realidad y las aplique en beneficio de su desarrollo personal.

Con todo lo escrito, ¿es posible hablar de una educación integral? En la práctica es probable que el concepto se vuelva un ideal, ya que difícilmente se abarcan todas las dimensiones de la persona en un solo proceso educativo. Sin embargo, una noción importante que se debe considerar es que la educación, si pretende ser integral, debe enfocarse en incidir en los conocimientos, las habilidades, las actitudes e incluso en la espiritualidad de los jóvenes. Claro está que para ello se debe echar mano de una diversidad de medios, algunos de ellos que son esenciales: el deporte, las artes, la convivencia entre educador y el joven, los contenidos, respondiendo a las expectativas de los jóvenes. Solo así puede esperarse que la educación genere impactos en la vida de los jóvenes más allá del aprendizaje de conceptos y contenidos. 